\title{
Assessment of eating behaviors in adolescents participating in regular physical activities
}

\author{
Dionysia Argyropoulou ${ }^{1}$, Argyrw Papadopetraki $^{2}$ and Maria Maridaki ${ }^{1}$ \\ ${ }^{1}$ Faculty of Physical Education and Sport Science, National and Kapodistrian University of Athens, Athens, Greece and \\ ${ }^{2}$ Medical School, National and Kapodistrian University of Athens, Athens, Greece
}

\section{Abstract}

Introduction: The combination of healthy food and fluid consumption is indicative for the nutritional habits of any individual. A balanced eating behavior is crucial during the growth phases, especially when they participate in regular physical activities in order to meet energy balance and nutritional quality.

Purpose: This study investigated the eating behaviors and fluid consumption in adolescents who participate regularly in various physical activities.

Methods: In this study, 218 adolescents, 110 males and 108 females, (age: $12.9 \pm 1.8$ yrs, height: $158 \pm 12.4 \mathrm{~cm}$, weight: $49.0 \pm 12.2 \mathrm{~kg}$, BMI: $19.4 \pm 2.8 \mathrm{~kg} / \mathrm{m}^{2}$ ) were recruited from Greece, participating in regular activities. Football, tennis, basket, swimming, track \& field, rhythmic gymnastics, tae kwon do and Greek traditional dance are the activities selected for intensive energy expenditure. A validated questionnaire was used for the identification of the nutritional attitudes and routine, while non-parametric statistics was utilized.

Results: The majority of participants (69.2\%) eat breakfast almost every day while the rest (30.8\%) doesn't eat at a regular basis. As it concerns the dairy products, $82.6 \%$ of the participants eat less than 2 portions per day. Furthermore, $43.1 \%$ of the participants seems to eat red meat at most 1 time per week while similarly, the $86.7 \%$ of them choose to consume fish only once. In terms of snack consumption, $81.6 \%$ of the adolescents, eat $1-2$ fruits per day, while in contrast, $53.6 \%$ of them are used to consume unhealthy sweets more than 3 times per week. Lastly, $54.5 \%$ of the participants drink less than 5 glasses water per day, whereas the $47.3 \%$ doesn't drink water at a regular basis during the training.

Conclusion: Our findings revealed that Greek adolescents' diet doesn't come to an agreement with the Healthy Lifestyle Pyramid. As far as it concerns the dairy products, which are important during the growth phases, subjects consume less than 2 portions per day rather than 4 which is the recommended. Also, they eat only once a week fish or red meat, while they prefer to consume unhealthy snacks frequently. Moreover, their fluid consumption habits don't meet their hydration needs even though they participate systematically in physical activities. Interestingly, although their eating behavior is not the appropriate, the BMI is within normal range, probably because of the adherence to regular exercise. However, the beneficial effect of exercise is not enough to stay healthy towards adulthood and they still need manipulation of their daily nutrition.

\section{Conflict of Interest}

There is no conflict of interest 